\title{
Issues facing the bioanalytical community: summary of round table discussions
}

First draft submitted: 21 July 2016; Accepted for publication: 8 September 2016; Published online: 19 September 2016

Keywords: new technologies • outsourcing • staff development

During the Tenth Workshop on Recent Issues in Bioanalysis (WRIB) meeting in Orlando, FL, USA (18-22 April 2016, [1]), Bioanalysis Zone organized an independent round table discussion, in which a number of bioanalytical experts from large pharmaceutical companies (Pharma) and contract research organizations (CROs) got together to discuss topical general issues that are facing the quantitative bioanalytical community. The round table discussion took place at the end of a long day of high-quality presentations, under the bright lights of a camera crew. The following is a summary of the discussions that took place and can be viewed in full on the Bioanalysis Zone website [2].

\section{Outsourcing strategies}

The CRO participants were keen to understand the outsourcing strategy of Pharma to help them put together their business plans. The consensus was that for small molecules, the bioanalytical work is considered more routine and hence has, to some extent, become commoditized. Hence, the strategy for most Pharma companies is to outsource the majority of small-molecule regulated preclinical and clinical work, with some degree of support from internal Pharma bioanalytical groups. However, for the increasing numbers of biopharmaceuticals and the novel constructs that are coming through different company pipelines, outsourcing by Pharma depends on the ability of CROs to invest in the equipment and staff required to support the analytical challenges and more complex assays that are arising from these molecules.

It was noted that smaller biopharmaceutical companies and large Pharma may have quite different requirements for bioanalytical outsourcing. Small companies may have little capability in this area and so are more reliant on the CROs for all aspects of method development, validation and study support, using them as a virtual laboratory. Whereas, larger Pharma are likely to have some inhouse bioanalytical capability that is capable of supporting some, or all, of these aspects. In addition, it was observed that smaller biopharmaceutical companies tend to place the support of a larger proportion of their earlyphase (discovery) studies out to contract. These studies often tend to be on the critical path for project progression and so the timelines for delivery of analytical data are often more aggressive than those for later development phase studies. There was also discussion around whether a large Pharma might have different approaches for outsourcing early discovery nonregulated work and later more regulated development work. It was stated that a lot of larger Pharma want to get their regulated work outsourced and to let CROs handle most aspects associated with that work. It is notable that large Pharma companies will invariably have bioanalytical monitors overseeing the outsourced work. There is a strong sense by Pharma that irrespective of where the work is being done, it is still a reflection of their company. Hence,
Neil Spooner ${ }^{* 1,2}$, Stephanie Cape $^{3}$, Roger Hayes ${ }^{4}$, Surinder Kaur ${ }^{5}$, John Kolman $^{6}$, Stephen Lowes ${ }^{7}$, Scott Summerfield ${ }^{8}$, Amanda Wilson ${ }^{9} \&$ Eric Woolf ${ }^{10}$

'Spooner Bioanalytical Solutions Ltd, Hertford, UK

${ }^{2}$ School of Life \& Medical Sciences, University of Hertfordshire, UK ${ }^{3}$ Bioanalytical Chemistry, Covance Laboratories, 3301 Kinsman Blvd, Madison, WI 53704, USA

${ }^{4}$ Laboratory Sciences, MPI Research, 54943 North Main Street, Mattawan, MI 49071, USA

${ }^{5}$ Genentech, 1 DNA Way, South San Francisco, CA 94080-4990, USA

${ }^{6}$ Translational Medicine, QPS, Delaware Technology Park, 1 Innovation Way, Newark, DE 19711, USA

${ }^{7}$ Bioanalytical \& ADME Laboratories, Q2 Solutions, 19 Brown Road, Ithaca, NY 14850, USA

${ }^{8}$ Bioanalysis, Immunogenicity \& Biomarkers, GlaxoSmithKline

Pharmaceuticals Research

\& Development, Park Road,

Ware SG12 ODP, UK

${ }^{9}$ Pre-Clinical Bioanalysis \& Toxicokinetics, Astra Zeneca, Building 623, Babraham Research Campus, Babraham, Cambridge CB22 3AT, UK

${ }^{10}$ Merck Research Labs, Department of Pharmacokinetics, Pharmacodynamics \& Drug Metabolism, West Point, PA 19486, USA

*Author for correspondence: neil@spoonerbioanalytical.co.uk 
For reprint erders, please contact reprints@future-science,com generated in-house. Conversely, smaller Pharma companies are more comfortable to rely on the CRO to deliver a regulatory approvable report in their format. Where large Pharma are outsourcing discovery work, they often use physically different CROs to those used for regulated later-phase work. This is often because the Pharma discovery and development groups are in different parts of their company's structures. Furthermore, the needs of the discovery and development Pharma groups are different. The choice of a CRO for the placement of work for regulatory submission will be heavily influenced by the CROs' reputation, size, history of compliance and successful regulatory inspections, whereas for discovery, the choice may be based upon the technical capability, flexibility and speed of the CRO laboratory.

For regulated bioanalytical work, the amount and type of work outsourced by Pharma differ between companies and are dependent on the stages of the drugdevelopment continuum. Although some outsource the whole package, including method development, validation and study support, the majority tend to develop and validate/qualify the bioanalytical method inhouse and then transfer it to the CRO. This is done either, because the CROs lack the required expertise with a particular molecular construct, or because the Pharma company prefers to have some indication of assay performance before handing the assay over to a CRO. Other Pharma companies prefer to develop the method and support a limited number of studies inhouse, to demonstrate the robustness of the method, before transferring the method to a CRO. In all cases, it is important to note that the overall accountability for the data when submitted for regulatory approval resides with the Pharma and not with the CRO. However, there is a shared responsibility between the Pharma and CRO to make sure that the right data are available.

Bioanalytical method development for biomolecule assays tends to take longer, and the clinical studies associated with them are often related to fewer samples than for small-molecule drug development. This changes the outsourcing paradigm and charging/cost basis for outsourcing this work, as a larger proportion of the cost is for method development and validation. This change requires communication and education of key stakeholders in Pharma, particularly procurement departments and groups receiving the final bioanalytical data, who might have false expectations based on previous experience with small-molecule drug-development programs.

How a CRO is selected varies widely between Pharma companies. Many use auctions to realize the best value for the Pharma company. In some cases, this process may be heavily led by procurement groups with minimal initial involvement of the company's own bioanalytical experts. In other cases, procurement may work from a short list put forward by the bioanalytical scientists. However, it is notable that the Pharma company's bioanalytical group generally has the last say on which CRO is used and that quality is the prime consideration for this selection, and not the cost alone. An important observation over the last 10 years has been the general change from cost to quality driving outsourcing decisions, with partnerships being a prime aspect of modern Pharma/CRO relationships. It was agreed that these partnerships should ideally involve scientist-to-scientist direct communication, to enable the efficient transfer of the method and subsequent troubleshooting. It was also expressed that it is very important that the client and CRO come together regularly to talk about not only the specific outsourced projects, but also to share new science, technologies and outputs from recent publications and scientific meetings. This contributes to everyone feeling part of a larger team and ensures that all are up to date with the latest innovations and regulatory guidance. An important aspect is that the CRO must be flexible to meet the inevitable fluctuations in workflow and timelines. The relationship also requires a good CRO project manager to oversee it. Furthermore, there is a requirement for consistency of all personnel involved throughout the lifetime of a project, as it is unlikely that the Pharma company will move a particular method between CROs during the long periods encountered for drug development. Although it may be difficult to guarantee stability of junior laboratory staff, most CROs work hard to retain key talent, and an active relationship with the Pharma client can help facilitate this, as it brings development opportunities for the CRO staff.

It was stressed that an important part of the Pharma/CRO relationship is to enable staff from either party to collaborate closely and if possible to work in each other's laboratories. This facilitates the introduction and transfer of new technologies and techniques and helps with troubleshooting issues that inevitably arise, as well as building stronger and more positive relationships between individual scientists and companies. It was also considered to be incredibly powerful for the Pharma company to share with the CRO not only general information about the clinical aspects of a particular project, but also the outcomes of an individual study and the impact to the patient(s). In turn, it is increasingly an expectation of the Pharma companies that the CRO bench staff understand the purpose for which the data generated from the samples, which they are analyzing, are being used. With the growth in biopharmaceu- 


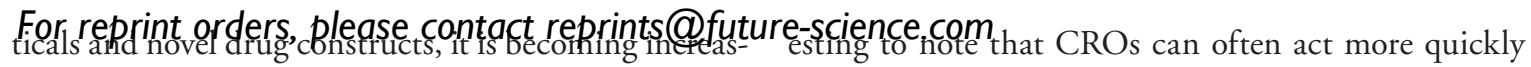
ingly important that $\mathrm{CRO}$ bench scientists have a broad understanding of drug development and the biology that underlies a particular project, particularly as the reliance of Pharma on CROs is increasing.

The panelists held the opinion that there is an increasing requirement and value in being able to work with CROs who offer an integrated approach, particularly for preclinical studies where the hand-offs between study directors and principle investigators for the in-life and bioanalytical aspects can become very complex, particularly when they are on different sites, or are part of different companies.

\section{Investment in new technologies}

There was a general perception from Pharma panelists that it is difficult for them to outsource the bioanalysis of new molecular constructs, or analyses involving novel workflows, particularly when investment in novel analytical approaches and equipment is required. In addition, it was observed that some CROs prefer to invest in more headcount rather than the implementation of routine sample-handling automation. The discussion confirmed that this was often the reality and that CROs are traditional followers, rather than leaders when it comes to the implementation of novel approaches. They tend not to have spare resource to invest in the research required to be an early adopter of new technologies. It was observed that Pharma clients often want workflows to be performed in a certain way, while CROs find it easier to make capital investments if they can be used across a broad range of clients, so technology has to be flexible. However, some Pharma companies are open to their suggestion and preferred workflows being used by other clients of the CRO. It was considered that this can be particularly beneficial in the discovery environment, where it can help drive down costs.

There was an understanding that implementing these new workflows offers the CROs an opportunity to differentiate themselves from other companies operating in the same space, but it was stressed that the technologies would have to be well researched first. It was observed that CROs' attendance at international meetings (such as WRIB) helps them to understand directions that Pharma are taking and what some of the emerging workflows and solutions might be. Furthermore, as the CROs work for multiple Pharma clients, they often tend to see new developments and approaches before many of their individual clients.

An opinion was given that it would be useful for the CROs to have at least a 6-month outlook on what might be coming in a client's portfolio, so that they can plan their workloads and investments. It was also interthan Pharma to invest in cost-effective solutions if the opportunity warrants.

One approach suggested to help bridge the gap in technology implementation was risk sharing between CROs and Pharma. This could take the form of dedicated production units at the CRO, giving priority access to a client in return for a guaranteed workload over an extended period. It was stated that instrument vendors often approach CROs offering them the opportunity to evaluate novel equipment. The advantage to the vendors is that the CROs generally require redundancy on instrumentation to cover downtime, and so it is likely that the CRO will invest in more than one unit of a particular technology. The equipment is also likely to be tested on a wide variety of analytes and a large number of samples, giving a more comprehensive evaluation than might be obtained elsewhere. An important observation was that CROs generally focus on investment to immediately increase efficiency with rapid payback, rather than making investments in new technology that may have a more speculative or longerterm payback period. They also require standardization to give flexibility between clients and assays, and to keep costs down.

Pharma often ask CROs to invest in the identical platform to that being used in the Pharma company's own laboratories. In these cases, there is generally very little leeway for the CRO to suggest alternative approaches, or instrumentation that might attain the same endpoint. Different Pharmas often ask for different approaches, so the CRO needs to consider the cost benefits carefully. There was some openness by Pharma to consider alternative approaches to achieve the same goal.

\section{Bioanalytical skills gap}

This part of the discussion centered on whether there is a growing skills gap for bioanalytical scientists in both Pharma and CROs. Whether the impact of such a gap is leading to difficulties in developing suitable approaches and innovative workflows for the analysis of novel construct molecules, including ease of assay transfer and technology between laboratories, were explored. Scientists with a strong chemistry and some biology background were traditionally sought by bioanalytical laboratories, where the drug entities could be defined as small-molecule white powders that could be analyzed routinely by simple sample preparation followed by LC-MS/MS. However, these skills are no longer considered to be enough now that Pharma are increasingly moving toward novel molecular constructs and modes of action. This requires an increasing understanding of biology and, particularly, immunology and protein chemistry, with routine LC-MS/MS no longer 
was. Furthermore, there is an increasing need to have staff who can interpret data, regardless of the analytical methodology used to generate it, particularly for regulated work. Hence, this broader skill set and the multifaceted approach to problem solving requires scientists with different backgrounds and skills to be brought together to look at problems in novel ways. The difficulty is in how to bring together these new groups, particularly when these staff are not readily available to hire. For these reasons, participants stated that they are now tending to recruit more $\mathrm{PhD}$ qualified biologists than they would have previously. However, these staff arrive with little, or no experience, of latest bioanalytical approaches (e.g., HRMS) and so require training in these techniques. Additionally, it was considered that graduate-level scientists are coming out of university with very few laboratory hours and fewer practical laboratory skills than may have been the case in the past. The participants also believed that the development of modern sensitive and simple-to-use MS instrumentation has been partially responsible for many existing staff having less understanding of the MS technologies that they routinely use, and the alternative experiments that are available (e.g., precursor and neutral loss scanning). One of the solutions put forward for this was to implement the cross training of staff. Bringing immunoassay and MS-based staff together in one place helps. Many companies are cross training and housing these groups closer than they did in the past, as a mixture of techniques and the agility to move between them is required for the challenges presented by novel molecular constructs.

Another solution to this issue is to recruit experienced staff. This is a particularly urgent need for CROs, as many of them are expanding rapidly and they need staff who can be effective immediately. Traditionally, this recruitment has been from Pharma companies undergoing reorganization. However, it was expressed that at the time of the discussion, this source has pretty much dried up, as there are currently less mergers in large Pharma. This creates a gap for the CROs, who are filling their vacancies by recruiting freshly qualified Bachelor- and PhD-level staff. However, these recruits tend to not have the skill required to become productive quickly. Hence, there is a training burden for the company, not only in MS, or immunoassay-based approaches, but also in general bioanalytical techniques and workplace skills. It was suggested that a good approach to help with this initial training burden is to get basic laboratory and bioanalysis skills training embedded in university teaching programs and work more with internships. However, this requires longer-term investment and planning, and most universities are more interested in academic research than teaching the fundamentals of analytical and bio- analytical science. One potential direction in the USA could be the use of Community Colleges who are more interested in vocational training. It was also stated that there are lessons to be learned from China and India, where their universities tend to be better at preparing their students for the world of industrial employment.

One observation was that many recruits at Bachelor level are coming into companies with an expectation that within 2 years they are ready to move on to nonlaboratory-based and managerial positions, resulting in high staff turnover with an outcome that there is a lack of base level bioanalytical skills in the group. It was considered that Pharma companies were more able to offer these recruits the career flexibility they were seeking, compared with CROs, as they were able to get their staff more broadly involved in drug development, rather than focusing just on regulated bioanalysis. Another aspect of the discussion around recruitment highlighted the difficulty in attracting $\mathrm{PhD}$-trained staff to perform regulated quantitative bioanalysis. The level of routine tasks and paperwork associated with regulated bioanalysis can often be a shock for candidates from a research background, resulting in poor motivation and retention issues. It was noted that increasing the use of automation can reduce the perception of routine for the bench level scientist and may therefore help with these retention issues.

It was stated that there is a growing realization that staff need to be trained, rather than recruited with the required skills already in place. This requires that Pharma and CRO companies make a longer-term investment in their staff and provide them opportunities to develop as bioanalysts and in broader aspects of drug development and scientific leadership, and reward them for success in these endeavors. This led to a discussion around possible routes forward for staff training. It was proposed that bioanalytical groups in Pharma and CROs could work together to develop industry-training courses, building on the success of current offerings from meetings, such as WRIB, AAPS and so on. Another idea was that CROs could offer a hands-on training service for other bioanalytical laboratories. This could be reciprocated with Pharma companies opening their doors to CRO staff to enable them to learn the broader aspects of drug development and where bioanalysis fits into the jigsaw puzzle.

\section{Conclusion}

This round table discussion brought together a number of passionate individuals who took part in a wide ranging and honest discussion on some of the current and upcoming issues facing the quantitative bioanalytical community. In particular, the discussion provided a platform for the participants to increase their under- 


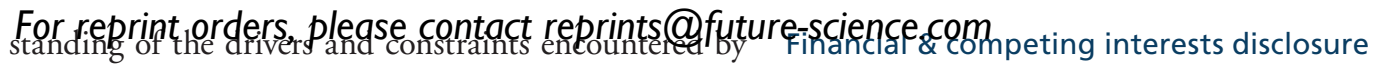

their Pharma/CRO partners in drug development. Furthermore, the participants were positive about working even more closely together in the future to benefit staff development and to build positive scientist-to-scientist relationships.

The videos of their discussions are, at the time of writing, one of the most viewed content on Bioanalysis Zone, which gives credence to the topics that were discussed being of high importance and relevance to this community and gives us renewed energy to continue to face the issues discussed and find workable solutions.

\section{Acknowledgements}

The authors would like to thank S Bishop, D March and S Nadarajah (employees of Future Science Group) and C Low (previous employee of Future Science group), for facilitating the round table discussion and helping to make it such a positive and enjoyable event.
$\mathrm{N}$ Spooner took part in and chaired the Panel Discussion in the capacity of Senior Editor of the journal Bioanalysis. All expenses for his trip were covered by Future Science Group. The authors have no other relevant affiliations or financial involvement with any organization or entity with a financial interest in or financial conflict with the subject matter or materials discussed in the manuscript apart from those disclosed.

No writing assistance was utilized in the production of this manuscript.

\section{References}

1 10th Workshop on Recent Issues in Bioanalysis (10th WRIB). Orlando, FL, USA, 18-22 April 2016.

2 Spooner N, Wilson A, Woolf E et al. Bioanalysis Zone panel discussion.

www.bioanalysis-zone.com 